\title{
Investigations and Simulations of All optical Switches in linear state Based on Photonic Crystal Directional Coupler
}

\author{
Sheler Maktoobi ${ }^{1}$, Rahim Ghayour ${ }^{2}$ \\ ${ }^{1,2}$ Islamic Azad University, Fars Science and Research Branch, Shiraz, Iran \\ E-mail: ${ }^{1}$ sh.maktoobi@yahoo.com, ${ }^{2}$ ghayour_r@hotmail.com
}

\begin{abstract}
Switching is a principle process in digital computers and signal processing systems. The growth of optical signal processing systems, draws particular attention to design of ultra-fast optical switches. In this paper, All Optical Switches in linear state Based On photonic crystal Directional coupler is analyzed and simulated. Among different methods, the finite difference time domain method (FDTD) is a preferable method and is used. We have studied the application of photonic crystal lattices, the physics of optical switching and photonic crystal Directional coupler. In this paper, Electric field intensity and the power output that are two factors to improve the switching performance and the device efficiency are investigated and simulated. All simulations are performed by COMSOL software.
\end{abstract}

Key words: optical switches, Optical directional coupler, photonic crystal, linear state

\section{Introduction}

Today, optical components, particularly the switches, has been considered in the telecommunication industry. Electrical techniques that cause delay and losses are used for switching operations, in many optical switches. Data transfer rate can be increased if switching operation is done in optical environment. All-optical methods and Photons crystals can improve efficiency of optical switches [1],[2]. Electronic switches are used to switch electrical signals that are controlled electromechanically by relay or electronically by semi-Conductors. Optical signals can be switched by electric switches, thus the optical signals are converted to electrical signals by photo detectors, and finally they are transformed optical signals by LED or laser. These transformations cause time delays and energy losses. Using all optical switches is one of the best ways to remedy this defect.

Optical switches are divided into two categories: the first category is optical switch and the second one is all optical switches. Modulation and deflection of light can be done by different methods, for instance mechanical, electrical, acoustic, magnetic method or optical control [3]. Nowadays, several technologies of switching are available and the number is being investigated [4],[5],[6]. In this paper, In order to reduce losses and delay of optical switches and increase high speed rate, All Optical Switches in linear state based on photonic crystal Directional coupler is designed and simulated by using COMSOL software.

Structure of this paper is in a way that will be further explained later. In following, the photonic crystal feature is presented. Then, all optical directional coupler is explained. Next, an all optical switch based on photonic crystal directional coupler is selected and finally the results have been simulated and analyzed. In order to simulations, COMSOL software was applied.

\section{Photonic Crystal Feature}

Today, Photonic Crystals appear in many areas of science, technology, medicine, and as a product of nature in the biological world. Photonic crystals are periodic optical nanostructures that affect the motion of photons in much the same way that ionic lattices affect electrons in solids. Photonic crystals occur in nature in the form of structural coloration and promise to be useful in different forms in a range of applications. Photonic crystals can be fabricated for one, two, or three dimensions [7],[8],[9]. One-dimensional photonic crystals can be either isotropic or anisotropic, with the latter having potential use as an optical switch [10]. Recently, a graphene-based Bragg grating (one-dimensional photonic crystal) has been fabricated and demonstrated its capability for excitation of surface electromagnetic waves in the periodic structure by using laser as the light source [11]. The Holey fiber or photonic crystal fiber can be made by taking cylindrical rods of glass in hexagonal lattice, and then heating and stretching them, the triangle-like air gaps between the glass rods become the holes that confine the modes[12].Photonic crystals are attractive optical materials for controlling and manipulating the flow of light.in Fig .1 a periodic structure from dielectric photonic crystal has also been shown. This triangular lattice is made of air holes in dielectric. 




Figure 1:A periodic structure from dielectric photonic crystal

\section{All Optical Directional Coupler}

All optical switches are used in order to reduce losses and high speed rate for this reason photonic crystals that play an important role are used. There are several kinds of all optical switches.in this paper one of the most important kinds is investigated and simulated. All optical directional coupler that is an all optical switch is composed of three region. The first region is input, the second one is coupling and the last region is output, as depicted in Fig 2. Radius of rods and central row radius are usually considered $0.2 \mathrm{a}$ and $0.14 \mathrm{a}$ respectively, where $\mathrm{a}$ is the structure lattice. The coupler is composed of two parallel waveguides by removing two rows of rods.in nonlinear state the central row has the most important role. Bending the waveguide separate coupling region from input and output region. The bend that is usually considered 60 to avoid the coupling and mixing of the output fields. Moreover the bent can reduce losses. Wave in a waveguide containing odd or even Super Mods are confined by discrepant phase. If the phase difference is an odd multiple of $\pi$, the wave will transmit to the next waveguide.

$\left(\mathrm{K}_{\text {even }}-\mathrm{K}_{\mathrm{odd}}\right) \mathrm{L}_{\mathrm{C}}=(2 \mathrm{n}+1) \pi$

In equation (1) $\mathrm{K}_{\text {odd }}$ and $\mathrm{K}_{\text {even }}$ are wave number are super modes respectively and $\mathrm{Lc}$ is length of the coupling region[13]

In linear state, which the refractive index of the central row is unchanged during the guidance of the optical wave so input optical wave, I1, with the proper frequency is guided to port $\mathrm{O} 1$.

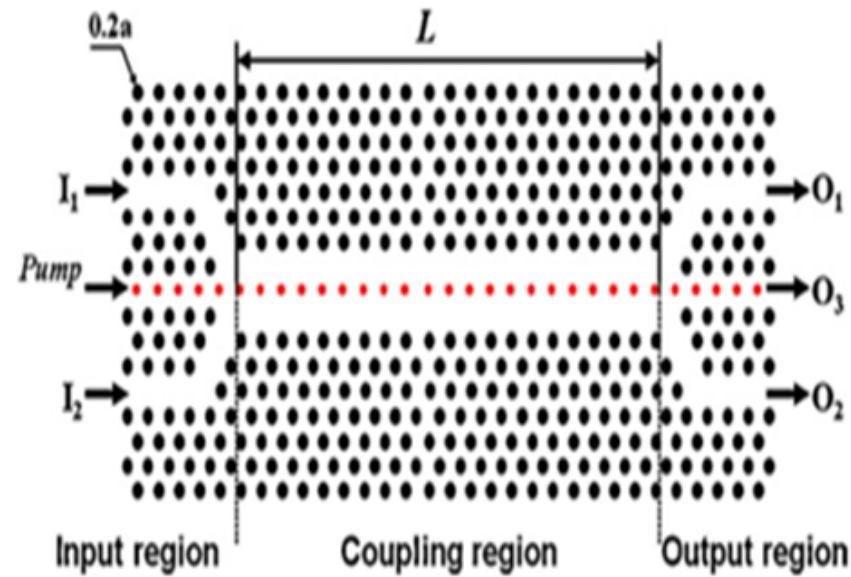

Figure 2 :A directional coupler switch based on Photonic crystal

\section{Simulation of All Optical Directional Coupler Switch}

\subsection{Simulation of Directional Coupler without Propagation}

In this paper, an all optical directional coupler based on photonic crystal analyzed and simulated. Our proposed structure is a rectangular $(31 \times 17)$ of air with an array of circular pillars of AlGaAs simulated by using COMSOL software and illustrated in Fig.3. Refractive index and radius of rods are 3.4 and $0.2 \mathrm{a}$ respectively, where a is the structure lattice constant. Central rods radius in directional coupler switch is usually considered $0.14 \mathrm{a}$ but in this paper we tried to obtain new results by changing the central rods radius [13]. A different structure with change in central row rods in fig. 3 are observed, the central rods radius is $0.13 \mathrm{a}$, a is the structure lattice constant in this paper, it is considered $575 \mathrm{~nm}$. This switch is designed where wavelength is $1550 \mathrm{~nm}$

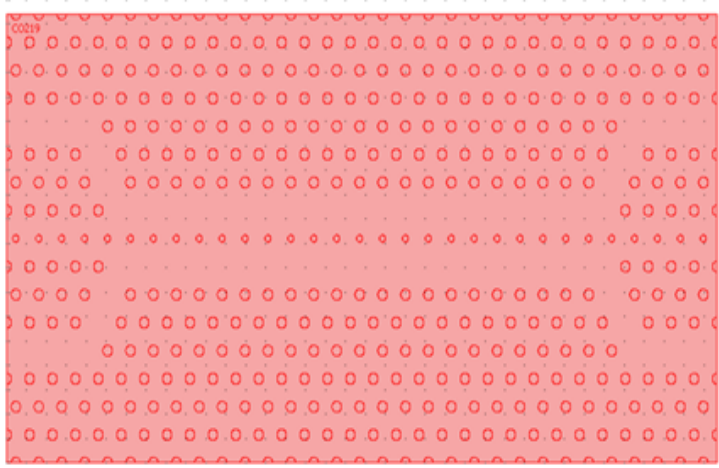

Figure 3 :Periodic structures of Photonic crystal without propagation 
The TM band gap of the structure, as depicted in Fig.4, is in the range of $0.2735 \leq \mathrm{a} / \lambda \leq 0.3833$, where $\lambda$ denotes the optical wavelength in free space. In fact, frequency range in order to analyze and simulate is determined. By removing some of the AlGaAs pillars in the structure of crystal, a guide for the frequencies within the band gap will be created

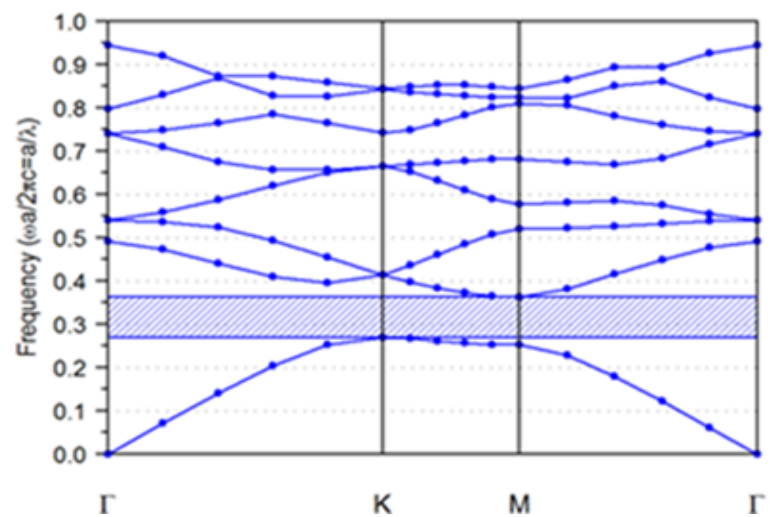

Figure4: Band diagram for TM polarization in a triangular lattice of $\mathrm{AlGaAs}$ rods

\subsection{FDTD (Finite Difference Time Domain) Simulation Basic}

While many electromagnetic simulation techniques are applied in the frequency-domain, FDTD (Finite Difference Time Domain) solves Maxwell's equations in the time domain. This means that the calculation of the electromagnetic field values progresses at discrete steps in time. One benefit of the time domain approach is that it gives broadband output from a single execution of the program; however, the main reason for using the FDTD approach is the excellent scaling performance of the method as the problem size grows. As the number of unknowns increases, the FDTD approach quickly outpaces other methods inefficiency [14].FDTD has also been identified as the preferred method for performing electromagnetic simulations for biological effects from wireless devices [15]. The FDTD method has been shown to be the most efficient approach and provides accurate results of the field penetration into biological tissues. In fact, the large-scale solution of Maxwell's equations for electromagnetic wave phenomena using FDTD and similar grid-based timedomain approaches may be fundamental to the advancement of electrical and computer engineering technology as we continue to push the envelope of the ultra-complex and the ultrafast. And their accurate modeling is essential to understand high-speed signal effects having wave-transport behavior [16].By associating many cell edges with materials, a geometrical structure can be formed within the FDTD grid such as the dielectric rectangular shown in Fig.5 (a), 5(b) by using COMSOL software. Each small part

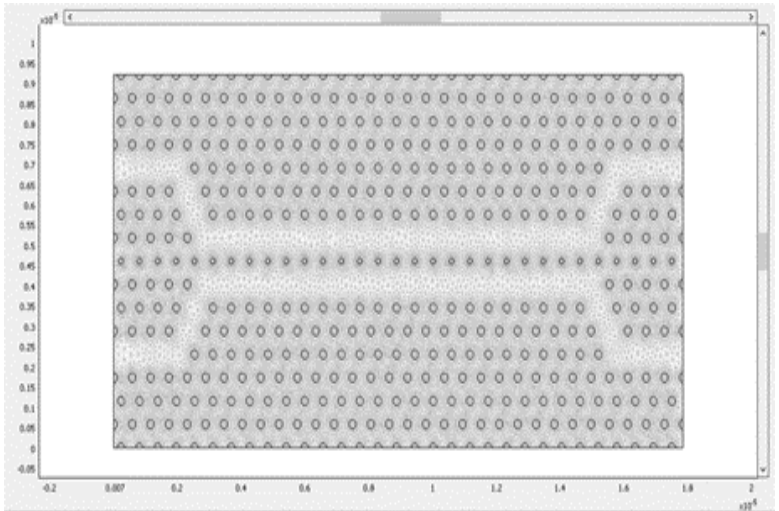

Figure 5 (a): Dielectric rectangular as meshed in an FDTD grid.

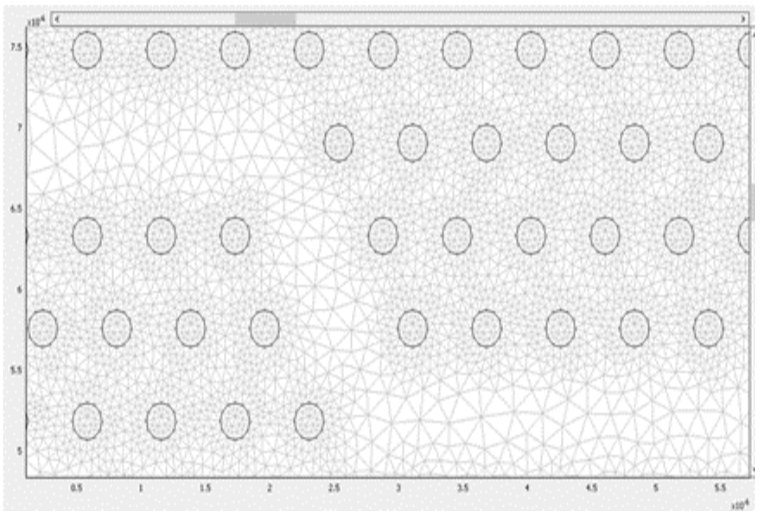

Figure 5 (b): Larger scale of a part of dielectric rectangular as meshed in an FDTD grid.

\subsection{Simulation of Photonic Crystal Directional Coupler}

An all optical switch in linear state based on photonic crystal was analyzed.in this structure central rods radius are considered $0.13 \mathrm{a}$, where a is structure lattice constant. In order to simulate aforementioned switch in linear state, light passes the next port.

Light, as depicted in Fig.6 can then propagate along the outlined guide geometry. It clearly shows the propagation of the wave through the guide in linear state. 


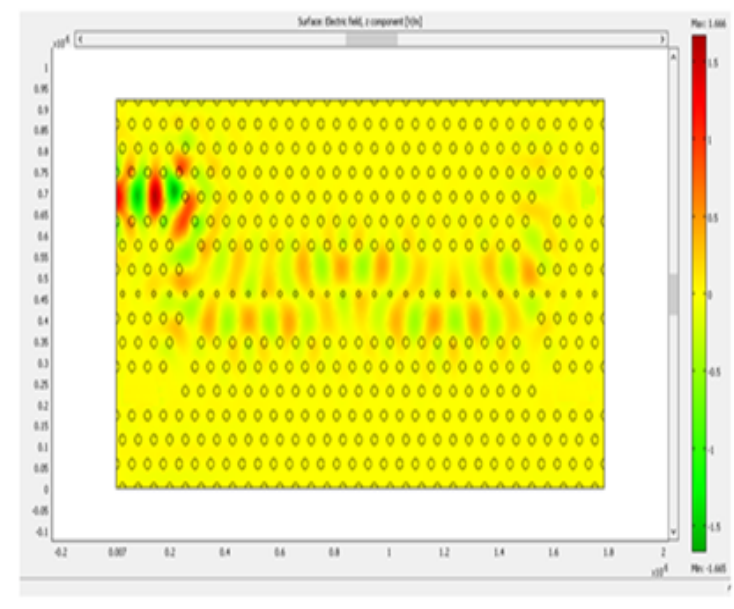

Figure 6: An all-optical switch in linear state

\subsection{The Results of All Optical Directional Coupler Switch}

Simulation and analysis of photonic crystal is done by using COMSOL software. By removing some of the AlGaAs pillars in the crystal structure a guide can be create for the frequencies within the band gap. Light can then propagate along the outlined guide geometry. So electric field can illustrate the propagation of the wave through the guide. Normalized electric field for our proposed structures is shown by Fig7.

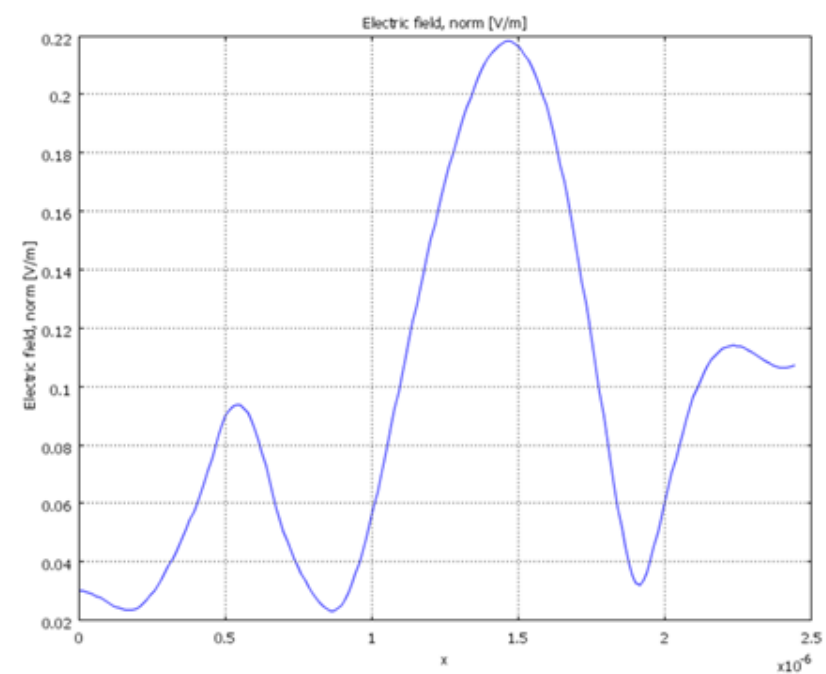

Figure7: Normalized electric field for our proposed structures

Some factors improve performance of devices, power output can evaluate Photonic crystal efficiency. Fig.8 contains a plot of normalized power flow where the highest and lowest power for the proposed structure in state linear are illustrated. One of the most important factors in designing and analyzing is output power and numerous applications are found by super flexible in power output.

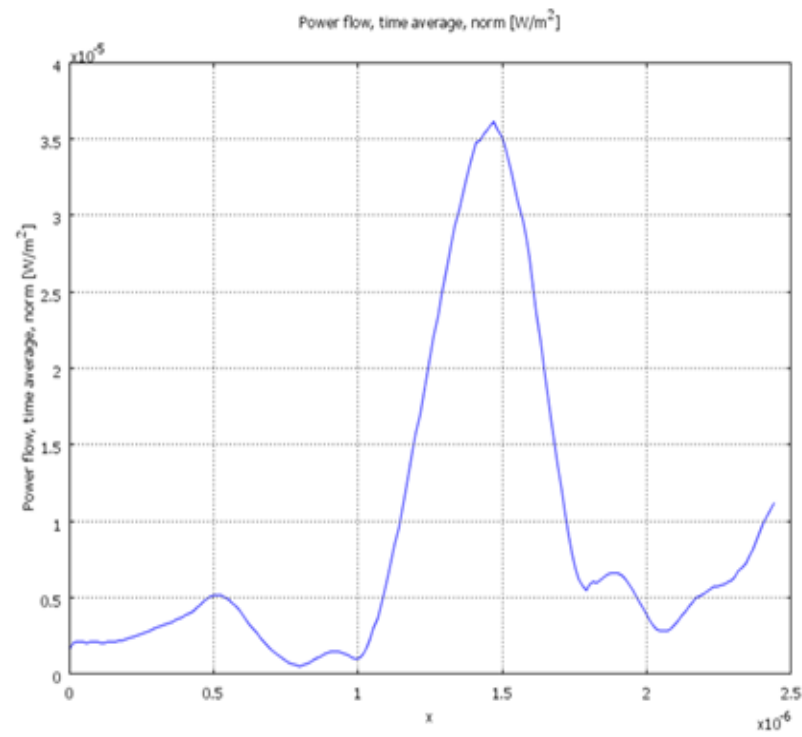

Figure8: Normalized output power

\section{Conclusion}

Electrical switches cause many delays and losses as well as a decrease in, speed and quality of switches. Nowadays, more advanced optical switch technologies were needed, particularly to remove the electrical conversion when switching light signals. In this paper, an all optical switch based on photonic crystal directional coupler in linear state has been proposed. The switching frequency is selected to have the most output power and the shortest coupling length in the linear state. This technology can be used to solve many significant problems as well as problems which are barely detectable in telecommunication such as bandwidth limitation, simultaneous transfer of limited information and low transfer speed.

\section{References}

[1] S.W. Leonard, J.P. Mondia, H.M. van Driel, O. Toader, S. John, K. Busch, A. Birner, U. Gosele, V. Lehmann, Tunable two-dimensional photonic crystals using liquid-crystal infiltration, Phys. Rev. B 61 (4) (2000) 2389-2392.

[2] T. Yasuda, Y. Tsuji, M. Koshiba, Tunable light propagation in photonic crystal coupler filled with 
liquid crystal, IEEE Photon. Technol. Lett. 17 (1) (2005) 55-57.

[3] Saleh, E.A., Teich, M.C., (1991).Fundamentals of photonics, John Wiley \& Sons, INC.

[4] C.M. Reinke, A. Jafarpour, B. Momeni, M. Soltani, S Khorasani, A. Adibi, Y. Xu, R.K. Lee, Nonlinear finite-difference time-domain method for the simulation of anisotropic, $\mathrm{x}(2)$, and $\mathrm{x}(3)$ optical effects, IEEE J. Lightwave Technol. 24 (1) (2006) 624-634

[5] A.T. Rahmati, N. Granpayeh, Design and simulation of a switch based on nonlinear directional coupler, Optik (in press).

[6] M.S. Saremi, M.M. Mirsalehi, Analysis of femtosecond optical pulse propagation in onedimensional nonlinear photonic crystals using finitedifference timedomain method, Optik 116 (10) (2005) 486-492.

[7] Huttunen, A., (2005). "Analysis and optimization of photonic crystal components for optical communications." Laboratory of computational Engineering, Helsinki University of technology.

[8] Johnson, S.G., (2005). "Photonic crystals: Periodic surprises in electromagnetism", $M I T$.

[9] Introduction to photonic crystals, single-period 1-D pcws

[10] Fiddy, Michael A.; Schenk, John O.; Cao, Yang (2008), Third Order Nonlinear Effect near a Degenerate Band Edge, Optics and Photonics Letters 1 (1): $1-7$.

[11] K.V.Sreekanth et al.; Zeng, Shuwen; Shang, Jingzhi; Yong, Ken-Tye; Yu, Ting (2012), "Excitation of surface electromagnetic waves in a graphene-based Bragg grating", Scientific Reports 2: 737.

[12] Frederic Zolla, Gilles, Renversez, Andre Nicolet. Foundations of photonic crystal fibers, MA 01923, USA.M. Ivanović, "Working one's way through LaTeX," Automatika, vol. 2009, no. 3-4, pp. 150-158, 2009.

[13] Eshaghi, A., Mirsalehi, M.M., Attari, A.R., Malek Abadi, S.A., (2008). "All-optical switching structure using nonlinear photonic crystal directional coupler", PIERS Proceedings: 587-591.

[14] Joseph, R.M., Taflove, A., (1997). “FDTD Maxwell's equations models for nonlinear electrodynamics and optics”, IEEE transaction antennas and propagation, Vol. 45, NO. 3:364-374.

[15] C95.3.2002, "Recommended Practice for Measurements and Computations with Respect to Human Exposure to Radio Frequency Electromagnetic Fields, $100 \mathrm{kHz}$ to $300 \mathrm{GHz}$," IEEE Standards and Coordinating Committee 28 on Non-Ionizing Radiation Hazards, April 2002.

[16] Jordan, A., Maple, C., (2004). "the modeling of the FDTD method based on graph theory", COMPEL: the international journal for computation and mathematics in electrical and electronic engineering, Vol. 23:694-700. 